\title{
Innovative Thought and Methodology of Planck Constant Experiment Based on Photoelectric Effect Theorem
}

\author{
Zheng-he SUN ${ }^{1}$, Yan-chao ZHANG ${ }^{2}$, Wen-jun LIU ${ }^{1, *}$ \\ Ying-ying $\mathrm{WANG}^{1}$ and Shou-tian REN ${ }^{1}$ \\ ${ }^{1}$ Department of Physics, Harbin Institute of Technology, Weihai, Shandong, China \\ ${ }^{2}$ Department of Information, Harbin Institute of Technology, Weihai, Shandong, China \\ ${ }^{*}$ Corresponding author
}

Keywords: Photoelectric Effect; Planck Constant; Experimental Methodology.

\begin{abstract}
This research aims to use an innovative thought and methodology to improve the teaching of college physics experiment. The detailed idea and methodology were introduced by a Planck Constant experiment based on Photoelectric Effect Theorem. In this experiment, a high precision measurement of Planck Constant was achieved by a self - made newly invented device. This device is self-developed with an open structure providing hands on measurement, which enables to conduct comparative trials with contrast tests. This new experimental design could inspire the students and help them develop creativity.
\end{abstract}

\section{Introduction}

\subsection{The Existing Problems in College Physics Experiment Teaching}

1. In the experiment of Planck constant measurements, mercury lamp filter was commonly equipped as a light source. However, there is one deficiency of this type of filter. The light through the filter is a combination of two or three spectral lines from mercury lamp light, which will derive a non-negligible measurement error of Planck Constant due to this non-monochromatic light [1]. This bias measurement parameter of Planck Constant measurement won't be able to echo Einstein's Photoelectric Effect Equation. Thus, a new methodology should be developed to improve this experiment.

2. This routine Planck Constant measurement experiment was out of date compare with modern science and technology. A new methodology needs to be developed to overcome the experimental limitation and optimize the results.

3. Some of the commercial experiment instruments are designed in black box, which are not intuitive for student to follow and understand the experimental details. These experimental devices are not well designed to inspire the students and help them develop creativity.

\subsection{Research Objectives}

This research is aim to improve the optimization of physics experiment outcomes by applying innovative methodology developed by modern technologies. The design methodology and idea could be used as guidance for other physics experiments to solve the existing design deficiencies and improve the routine experimental designs.

\section{Methodology and Results}

In this paper, the measurement of Planck's constant based on Photoelectric Effect Theorem is demonstrated as a case study to solve existing experimental deficiencies.

\subsection{Experiment Methodology}

The measurement of the Planck's constant experiment is based on the principle of Einstein's Photoelectric Effect Equation. In this research, we found the experiment results are not satisfied the 
Einstein's Photoelectric Effect Equation when the incident light contains multiple monochromatic lights [1]. This experimental error is induced by the spectral linewidth of monochromatic light and other interference light. Our experiment also proves that a better monochromaticity light source could achieve a higher measurement accuracy of Planck constant. This conclusion can be significantly contributed to experiment instrument design, fabrication, optimizing the measurement error and textbooks revision.

\subsection{Improvement of Experiment Methodology}

The original mercury light filter with narrow band filtering method could provide a Planck constant measurement of $7.225 \times 10-34 \mathrm{~J} \cdot \mathrm{S}$ on average, which is almost $9 \%$ deviation from the accepted Planck constant value $\left(6.626 \times 10^{-34} \mathrm{~J} \cdot \mathrm{S}\right)$ [2]. After comparing multiple spectral analyses with monochromatic light such as mercury lamps and semiconductor lasers, we found laser is a more ideally light source given its superior monochromaticity [1]. The Planck constant experiment based on our self-made instrument equipped with semiconductor laser source could improve the measurement accuracy with a relative measurement error $0.02 \%$ [1]. This measurement error is much smaller compared with the measurement obtained by original method above. Per experiment results, this measurement error will be significantly increased when laser light scattered by the bare metal or the plastic materials before entering the photocell. That's because the monochromaticity of the incident light was weakened by the fluorescence from scattering substances. One key technology of our instrument is the design of the light source position. By setting the laser device close to the photocell, the laser can be close-range, barrier-free, light-shielded and stable. Besides, the metal parts are all blackened in order to maintain the monochromaticity of incident light. An accurate Planck constant measurement could be achieved by properly selecting the light intensity to screen out the weak spectral lines beyond the selected standard wavelength.

\subsection{Improvement of Experiment Results}

Development of New Photoelectric Effect Experiment System. There are many advantages of using small semiconductor lasers as a light source. First of all, it could enhance the instrument performance; reduce the instrument size and weight; cut down the cost of production; provide high efficiency and precision with low energy consumption. These good characteristics could overcome the deficiency of routine Planck constant experiment. Secondly, the students could develop their hands-on ability by operating the instrument such as replacing lasers devices or connecting wires and so on. Their understanding of physics concepts could also be enhanced by directly comparing difference light sources with the corresponding measurement results.

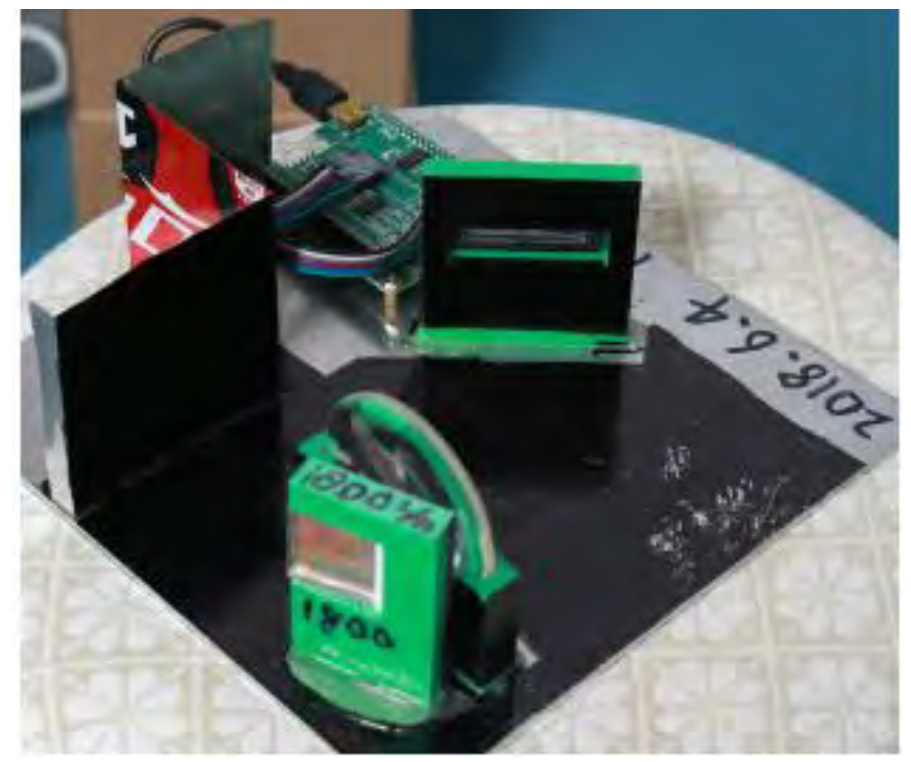

Figure 1. Intuitive Open Structure Spectrometer 
Introduction of Experiment Instrument: Spectrometer. In this study, the Planck constant experimental system is constructed by combining the photoelectric effect system with our self-made spectrometer, which could provide a laser wavelength measurement with confidence interval half width less than $0.1 \mathrm{~nm}$ [3]. This spectrometer is equipped with a planar CCD camera to record the spectral lines, while transferring the data into a computer to display the spectral curve. As shown in Figure 1, the spectrometer featured with a light splitting device based on holographic or reflective grating and obtained a simple, intuitive open structure compared with the old expensive ones designed in black box. Combined with programming, the mercury lamp is used to calibrate the spectrometer, which could enrich the experimental content and improve the experimental quality.

\subsection{The Content of Comparative Experiment Teaching}

Our self-invented instrument adds a new experimental method to the measurement of Planck constant, which enables us to carry out a more comprehensive research experiment. This experiment could combine different light sources and different methods of measuring optical frequency to help student develop their divergent thinking and creativity. The experimental class focuses on teaching a variety of methods and instrumental principles. Adding some experimental contents using comparative methods, analyzing the causes of differences and chasing the roots of ideas would help students better understand the experimental mechanisms.

\section{Discussion and Conclusion}

This research first discovered and investigated the mechanisms of Plank constant measurement error. Then improved the experiment methodology based on Physics principles, which could not only enhance the experimental efficiency but also reduce the experiment relative measurement error from $9 \%$ to $0.02 \%$.

This project found the measurement parameter of Planck constant based on multiple monochromatic incident lights do not accurately satisfy the principle of Einstein's Photoelectric Effect Equation. Besides, the measurement accuracy could be increased by improving the monochromic purity of incident light.

After comparing multiple light sources by spectral analyses, the semiconductor laser was used as our instrument light source given its superior monochromaticity. In our self-developed instrument, the laser light source position was carefully designed to prevent its monochromaticity from being weakened. Furthermore, the light intensity was also properly reduced in order to screen out the weak spectral lines other than the monochromatic light. In the end, the high-precision measurement of laser wavelength by spectrometer could ensure the data accuracy for parameter calculations. Therefore, our spectrometer with intuitive open-structure design could overcome the deficiencies from the original spectrometer with black box design.

Moreover, this methodology could be combined with the original Plank constant measurement experiment as additional experiment content. This combination could provide comparisons between difference study methods to analyze the causes of differences and chase the roots of principle to understand the experimental mechanism. Therefore, we can obtain a new methodology which could be generally applicable to solve the existing deficiencies and guide other experimental projects. In addition, the experiences and methods acquired from this project could be used as a model for other experimental and lab constructions.

The Einstein Photoelectric Effect equation was only theoretically. A future research can be conducted to further investigate the mechanisms of Plank constant measurement error in order to fully develop a mathematical formula to do the error correction. This mathematical analytic formula of the corrected error should be based on the modification of the Einstein Photoelectric Effect equation. Then we can apply this 'Practical Einstein photoelectric effect equation' in physical experiments. Regardless of the spectral linewidth of monochromatic incident light or the components of the mixed 
spectral lines from incident light, we can always correct the measurement error by plugging these variables into formula to derive Plank constant.

\section{Acknowledgement and Grant Support}

This research was financially supported by the National Collogue Physics Experimental Education Foundation (Innovative Thought and Methodology of Planck Constant Experiment based on Photoelectric Effect Theorem), Project Number: 01-201601-52.

\section{References}

[1] SUN Zheng-he, LU Te-an, LIU Tian-yu, LONG Yong-ji, An experimental new method adopting small semiconductor laser weak light source for measuring the Planck constant based on the photoelectric effect [J]. University physics China, 2017, 36 (1): 28-32

[2] ZHANG Kai-xiao, KONG Wei-yu, ZHU Wei-hua, SONG Jian-ping, LIU Min-yi, Error analysis and correction of the Planck constant measured by external photoelectric effect [J]. Physics Experiment China, 2013, 33 (1): 40-42

[3] LONG Yong-ji, TIAN Yu-chen, LIU Wen-jun, SUN Zheng-he, The development of simple spectrometer for experimental teaching [J]. Physics Experiment China, 2018, 38 (2): 52-56 